\section{Metabolische Vorteste}

G. F. Hoffmann ${ }^{1}$, C.-D. Langhans ${ }^{2}$ und A. Schulze ${ }^{3}$

${ }^{1}$ Zentrum für Kinder- und Jugendmedizin,

Universitätsklinikum Heidelberg, Heidelberg, Deutschland

${ }^{2}$ Stoffwechsellabor - GCMS, Universitätsklinik für Kinder-

und Jugendmedizin, Heidelberg, Deutschland

${ }^{3}$ Clinical and Metabolic Genetics The Hospital for Sick

Children, University of Toronto, Toronto, Kanada

Synonym(e) Gruppenreaktionen

Beschreibung Im selektiven Stoffwechselscreening können wichtige diagnostische Hinweise aus einfachen Voruntersuchungen im Urin gewonnen werden. Zu diesen Spottesten gehören verschiedene qualitative und halbquantitative Methoden meist in Form von Tüpfelproben oder Sticktests.
Gebräuchlich sind der D Dinitrophenylhydrazin-Test (DNPH-Test) zum Nachweis von Ketoverbindungen (z. B. verzweigtkettige Oxosäuren bei der Ahornsiruperkrankung), die Reduktionsprobe ( $\vee$ Reduktionsproben) auf reduzierende Substanzen (Zucker), die Brand'sche Probe ( $\triangleright$ Brand-Test, Nitroprussid-Test) zum Nachweis von schwefelhaltigen Aminosäuren (Cystein, Homocystein) und der $>$ Sulfit-Test zur halbquantitativen Bestimmung von Sulfitionen (Diagnostik des Sulfitoxidasemangels).

\section{Literatur}

Gibson KM, Duran M (2014) Simple tests. In: Blau N, Duran M, Gibson KM, Dionisi-Vici C (Hrsg) Physician's guide to the diagnosis, treatment, and follow-up of inherited metabolic diseases. Springer, Berlin/Heidelberg

Zschocke J, Hoffmann GF (2012) Vademecum Metabolicum - Diagnose und Therapie erblicher Stoffwechselkrankheiten, 4. Aufl. MilupaSchattauer Verlag, Stuttgart 